\title{
PSMA-Targeted Radionuclide Therapy and Salivary Gland Toxicity: Why Does It Matter?
}

\author{
David Taïeb ${ }^{1}$, Jean-Marc Foletti ${ }^{2}$, Manuel Bardiès ${ }^{3,4}$, Palma Rocchi ${ }^{5}$, Rodney J. Hicks ${ }^{6}$, and Uwe Haberkorn ${ }^{7,8}$ \\ ${ }^{1}$ Department of Nuclear Medicine, La Timone University Hospital, CERIMED, Aix-Marseille University, Marseille, France; ${ }^{2}$ Aix- \\ Marseille University, APHM, IFSTTAR, LBA, Hôpital de la Conception, Chirurgie Maxillo-Faciale, Marseille, France; ${ }^{3}$ Inserm, \\ UMR1037, CRCT, Toulouse, France; ${ }^{4}$ Université Toulouse III-Paul Sabatier, UMR1037, CRCT, Toulouse, France; ${ }^{5}$ Inserm, \\ UMR1068, CRCM, Aix-Marseille University, Marseille, France; ${ }^{6}$ Sir Peter MacCallum Department of Oncology, University of \\ Melbourne, Melbourne, Victoria, Australia; ${ }^{7}$ Department of Nuclear Medicine, University Hospital Heidelberg, Heidelberg, \\ Germany; and ${ }^{8}$ Clinical Cooperation Unit Nuclear Medicine, German Cancer Research Center (DKFZ), Heidelberg, Germany
}

$\mathbf{T}$ he promise of prostate-specific membrane antigen (PSMA)targeted radionuclide therapy is being demonstrated by a growing number of reports detailing institutional experience with various agents, and prospective clinical trials are in progress to further establish the safety and efficacy of this approach in advanced, castrate-resistant prostate cancer. Although extremely promising, PSMA-ligand therapy remains a noncurative treatment, and therefore, the prolongation of survival and amelioration of diseaserelated symptoms must be balanced against the direct toxicities of the treatment and their impact on quality of life. Of these, xerostomia is among the most common and debilitating, particularly for ${ }^{225}$ Ac-PSMA. The nature of this dysfunction is incompletely understood, and strategies for its prevention and treatment are still under evaluation.

The salivary glands are divided into major (parotid, submandibular, and sublingual glands) and accessory or minor groups. The amount of saliva secreted by the salivary glands is approximately 1 $\mathrm{L} / \mathrm{d}$ (70\% arising from the major salivary glands). The salivary $\mathrm{pH}$ ranges from 6.5 to 7 . Saliva has complex functions that act together to inhibit oral bacterial overgrowth and to protect dentition. Although salivary compounds have been fully characterized, saliva still remains impossible to synthetize. Radiotherapy-induced xerostomia is multifactorial. The primary cause of irreversible hyposalivation is loss or impairment of acinar cells and their progressive replacement by connective tissue and fibrosis. The mechanisms involved are both the loss of functional glandular stem cells or progenitor cells and the impairment of microvasculature and parasympathetic innervation. Below the threshold of $50 \mathrm{~Gy}$, severe dysfunction to gland tissue is rare, and radiation damage is generally transient and reversible. In irradiated patients, the saliva becomes more viscous and more acidic, which compromises its antibacterial action. Salivary hypofunction is the objective decline in salivary flow, whereas xerostomia is the subjective perception of dry mouth by the patient. Hyposalivation and its corollary xerostomia are common $(68 \%-91 \%)$ in long-term

Received Jan. 9, 2018; revision accepted Jan. 9, 2018.

For correspondence or reprints contact: David Taïeb, Biophysics and Nuclear Medecine, La Timone University Hospital, European Center for Research in Medical Imaging, Aix-Marseille University, 264, rue SaintPierre, 13385 Marseille, France.

E-mail: david.taieb@ap-hm.fr

Published online Feb. 9, 2018

COPYRIGHT (c) 2018 by the Society of Nuclear Medicine and Molecular Imaging. DOI: 10.2967/jnumed.118.207993 irradiated patients with head and neck cancer. Hyposalivation exacerbates tooth decay and periodontal disease. Irradiated patients complain about problems with mastication, swallowing, sleep, and speech; a burning sensation in the mouth; and dysgeusia. Taken together, these symptoms severely impair the quality of life of patients. The morbidity of irradiation related to salivary gland dysfunction can be scored with specific grading systems developed by the Radiation Therapy Oncology Group.

Similar side effects may be obtained by internal radiotherapy with small molecules targeting the PSMA, which are being used for therapy of metastasized prostate cancer. Although immunohistochemistry shows a rather heterogeneous PSMA staining with only low to moderate intensity, the uptake of radiolabeled smallmolecule-based PSMA ligands is high, suggesting both specific and nonspecific tracer accumulation, with the mechanism of the latter remaining unclear. The frequency and extent of the resulting symptoms are dependent on the absorbed dose and the isotope used. In contrast to small molecules, radiolabeled anti-PSMA antibodies show only low uptake in the salivary glands (1), supporting the hypothesis that the accumulation of small-molecule inhibitors of PSMA is at least partially nonspecific.

A first iteration of PSMA-directed radionuclide therapy of advanced, castration-resistant prostate cancer used ${ }^{131}$ I. That study involved 28 patients and took place in the era before abiraterone and enzalutamide. Seven of these 28 patients treated with a single administration of ${ }^{131}$ I-labeled MIP1095 reported a slight to moderate xerostomia, and in 1 patient, mucositis was detected. These side effects recovered after 3-4 wk (2). Multiple administrations ( $\leq 3$ fractions) were considered in a further 34 patients. In this analysis, xerostomia higher than grade 1 occurred more frequently in patients receiving a higher number of fractions. Again, most patients reported recovery from xerostomia after a few weeks. However, the duration of the symptoms was longer after the second or third therapy in most cases (3). Data for xerostomia after therapy with ${ }^{177} \mathrm{Lu}$-labeled PSMA617 are available from different groups. In an analysis of 30 patients treated with 3 cycles of $6 \mathrm{GBq}$, most of the patients reported no relevant dysfunction of the salivary glands (4). Substitution of saliva (spray or gel) was prescribed for 2 patients who developed xerostomia after the third cycle. After the first and second cycles, only transient xerostomia was occasionally observed. Similar data were obtained from a multicenter analysis of 145 patients, with mild to moderate xerostomia reported for only 11 patients (8\%) (5). It can 
thus be concluded that at therapeutically relevant activities, ${ }^{177} \mathrm{Lu}-$ labeled PSMA rarely leads to symptoms. In contrast, although leading to excellent therapeutic effects-even with complete remissions in a considerable number of cases — ${ }^{225} \mathrm{Ac}$ may also lead to destruction of the salivary glands (6). This seems to be a function of the activity administered to the patient. Treatment activities of $50 \mathrm{kBq} / \mathrm{kg}$ were without toxicity but induced an insufficient antitumor response in patients with a high tumor burden. However, an increase in administered activity led to severe xerostomia, which became the dose-limiting toxicity if treatment activity exceeded $100 \mathrm{kBq} / \mathrm{kg}$ per cycle (6). From a dosimetry standpoint, there is wide interpatient variability both in tumors and in healthy tissues, including the salivary glands. The range of absorbed dose delivered to the salivary glands per administered activity of ${ }^{177} \mathrm{Lu}$-DKFZ-PSMA-617 varied from 0.8 to $2.5 \mathrm{~Gy} / \mathrm{GBq}(4,7,8)$. Regardless of the methodology implemented, all approaches relied on planar imaging and used an OLINDA sphere module to evaluate the absorbed dose delivered to salivary or parotid glands. In a recent article, Kratochwil et al. reported dosimetric estimates for ${ }^{225}$ Ac-PSMA-617 based on extrapolation of the pharmacokinetics obtained with ${ }^{177} \mathrm{Lu}$-PSMA617 reported in their previous article (6). Substitution of an $\alpha$-emitter $\left({ }^{225} \mathrm{Ac}\right)$ for a $\beta$-emitter (such as ${ }^{177} \mathrm{Lu}$ ) has dramatic dosimetric implications, with huge absorbed dose gradients.

Preventive strategies have not been successful in mitigating these side effects of salivary gland function (local cooling, lemon juice and vitamin $\mathrm{C}$, or a displacement strategy using PMPA [2(phosphonomethyl)pentane-1,5-dioic acid]) (9,10). Accordingly, other than developing ligands with lower salivary gland uptake, prospects for preventing salivary gland radiotoxicity appear limited, except by reducing the administered activity, which may compromise therapeutic efficacy. However, it may be that higher administered activities are achievable with the first cycle of treatment when tumor burden and PSMA-binding affinity are highest and provide the protective benefit of a tumor-sink effect on salivary uptake. Alternatively, radioprotectors, such as amifostine, could be considered but may also reduce radiosensitivity in tumor sites.

Accepting that some degree of salivary gland dysfunction is likely, therapeutic interventions to reduce its impact on quality of life become relevant. The first-line treatment for radiotherapy-induced salivary gland dysfunction in patients with head and neck cancer is the use of sialagogues, namely pilocarpine and cevimeline, with proven efficacy in all stages of hyposalivation. However, as muscarinic receptor agonists, their use is limited by poorly tolerated side effects. On the other hand, salivary substitutes and mouthwash have no proven effect on hyposalivation but can temporarily relieve xerostomia symptoms. It is likely that these approaches will be similarly effective in the setting of PSMA-related salivary gland toxicity.

Future prospects for salivary gland preservation may include intraglandular injection of botulinum toxin or compounds such as vitamin $\mathrm{E}$ and $\mathrm{MnBuOE}(\mathrm{Mn}(\mathrm{III})$ meso-tetrakis( $N$-n-butoxyethylpyridinium-2-yl)porphyrin), as well as sialendoscopy, which has shown clinical improvement in patients with radioiodideinduced sialadenitis. The most promising approach to addressing the problem of radiation-induced hyposalivation appears likely to be therapies that lead to regeneration of salivary tissue, including intraglandular gene therapy (e.g., aquaporin 1, Sonic hedgehog), downregulation of key regulators of DNA damage-induced apoptosis (antisense therapy), and stem cell therapy.
At present, ${ }^{177} \mathrm{Lu}$ - and ${ }^{225} \mathrm{Ac}$-labeled PSMA ligands are used predominantly after failure of all guideline-conforming therapies, and therefore treated patients usually have a limited life expectancy, which offsets the duration of possible side effects. Nevertheless, accumulating evidence suggests that stratification of risk of salivary gland toxicity balanced against the likelihood of therapeutic benefit is needed to select the appropriate patients for treatment, the optimal isotope, and the appropriate administered activity. Introduction of $\alpha$-therapy earlier in the course of the disease should be done in the setting of prospective studies because a reduction of administered activity may be needed. However, a reduction of administered activity may limit therapeutic efficacy, which is a strong argument for a controlled systematic assessment of the therapeutic window.

Attempts to prevent xerostomia have been largely unsuccessful, and therefore more work is required to optimize administered activity in order to balance the risk of salivary toxicity against therapeutic effectiveness. Improved prospective radiation dosimetry models will need to be developed. Efforts to further improve our understanding of the mechanisms of nonspecific uptake of PSMA ligands in the salivary glands may lead to new preventive strategies, whereas improved treatments of salivary gland dysfunction, if these can be identified, are also important. We await the results of ongoing gene therapy trials with interest.

\section{DISCLOSURE}

No potential conflict of interest relevant to this article was reported.

\section{REFERENCES}

1. Tagawa ST, Milowsky MI, Morris M, et al. Phase II study of lutetium-177labeled anti-prostate-specific membrane antigen monoclonal antibody J591 for metastatic castration-resistant prostate cancer. Clin Cancer Res. 2013;19:51825191.

2. Zechmann CM, Afshar-Oromieh A, Armor T, et al. Radiation dosimetry and first therapy results with a ${ }^{124} \mathrm{I} /{ }^{131} \mathrm{I}$-labeled small molecule (MIP-1095) targeting PSMA for prostate cancer therapy. Eur J Nucl Med Mol Imaging. 2014;41: 1280-1292.

3. Afshar-Oromieh A, Haberkorn U, Zechmann C, et al. Repeated PSMA-targeting radioligand therapy of metastatic prostate cancer with ${ }^{131}$ I-MIP-1095. Eur J Nucl Med Mol Imaging. 2017;44:950-959.

4. Kratochwil C, Giesel FL, Stefanova M, et al. PSMA-targeted radionuclide therapy of metastatic castration-resistant prostate cancer with ${ }^{177} \mathrm{Lu}$-labeled PSMA617. J Nucl Med. 2016;57:1170-1176.

5. Rahbar K, Ahmadzadehfar H, Kratochwil C, et al. German multicenter study investigating ${ }^{177} \mathrm{Lu}-\mathrm{PSMA}-617$ radioligand therapy in advanced prostate cancer patients. J Nucl Med. 2017;58:85-90.

6. Kratochwil C, Bruchertseifer F, Rathke H, et al. Targeted alpha-therapy of metastatic castration-resistant prostate cancer with ${ }^{225}$ Ac-PSMA-617: dosimetry estimate and empiric dose finding. J Nucl Med. 2017;58:1624-1631.

7. Delker A, Fendler WP, Kratochwil C, et al. Dosimetry for ${ }^{177}$ Lu-DKFZ-PSMA617: a new radiopharmaceutical for the treatment of metastatic prostate cancer. Eur J Nucl Med Mol Imaging. 2016;43:42-51.

8. Yadav MP, Ballal S, Tripathi M, et al. Post-therapeutic dosimetry of ${ }^{177} \mathrm{Lu}-\mathrm{DKFZ}$ PSMA-617 in the treatment of patients with metastatic castration-resistant prostate cancer. Nucl Med Commun. 2017;38:91-98.

9. Afshar-Oromieh A, Avtzi E, Giesel FL, et al. The diagnostic value of PET/CT imaging with the ${ }^{68} \mathrm{Ga}$-labelled PSMA ligand HBED-CC in the diagnosis of recurrent prostate cancer. Eur J Nucl Med Mol Imaging. 2015;42:197-209.

10. Kratochwil C, Giesel FL, Leotta K, et al. PMPA for nephroprotection in PSMA-targeted radionuclide therapy of prostate cancer. J Nucl Med. 2015;56: 293-298. 